\title{
Diazepam attenuates conditioned odor potentiation of startle in rats
}

\author{
ALEX VISHNEY and RICK RICHARDSON \\ University of New South Wales, Sydney, New South Wales, Australia
}

\begin{abstract}
Recently, Richardson, Vishney, and Lee (1999) described a simple protocol whereby conditioned odor potentiation of startle (OPS) could be observed in rats. The aim of the present study was to extend their findings by determining whether OPS is attenuated by pretest intraperitoneal injection of diazepam. The results showed that diazepam $(0.6 \mathrm{mg} / \mathrm{kg})$ markedly attenuated OPS at test (Experiment 1) and that this attenuation was not due to diazepam's affecting either odor detection or mobility (Experiment 2). The results suggest not only that the OPS effect is mediated by fear, but also that it may be mediated by the same neural substrates that mediate conditioned tone and conditioned light potentiated startle.
\end{abstract}

In the presence of a conditioned stimulus (CS) that has previously been paired with shock, rats display a greater startle response to a loud, sudden noise than they do in the absence of the CS. This enhanced amplitude of the startle response is taken as evidence that the CS elicits a central state of fear in the animal (Davis, 1992), hence the term fear potentiated startle (FPS). The idea that FPS is mediated by fear is well supported empirically. For instance, FPS correlates well with other measures of fear, such as freezing (Leaton \& Borszcz, 1985) and is attenuated by pretest administration of a number of clinically used anxiolytic drugs ( for a review of drug effects on FPS, see Davis, Falls, Campeau, \& Kim, 1993).

One of the most productive uses of the FPS preparation has been in the examination of the neural structures involved in conditioned emotional responses. Indeed, research employing FPS, as well as other fear indices, has lead to the development of a number of models of the neural substrates of conditioned fear (e.g., Charney, Grillon, \& Bremner, 1998; Davis, 1992; LeDoux, 1993). Though these models are well supported empirically, they share an important omission. Specifically, all of the studies that have contributed to their development have employed either visual or auditory CSs and, as a result, little is known of the neural substrates of conditioned olfactory fear.

As an initial step to address this issue, we recently developed a simple protocol whereby conditioned odor potentiation of startle (OPS) can be observed (Richardson, Vishney, \& Lee, 1999). In the first experiment of that

This research was supported by grants from the Australian Research Council to R.R. and an Australian Postgraduate Award Scholarship to A.V. The authors acknowledge the helpful comments of Fred Westbrook on an earlier draft. Address correspondence to A. Vishney or R. Richardson, School of Psychology, Faculty of Life Sciences, The University of New South Wales, UNSW Sydney, 2052, Australia (e-mail: r.richardson@unsw.edu.au). study, rats given odor-shock pairings at training showed potentiated startle when the odor was presented at test, whereas a group given explicitly unpaired presentations of odor and shock at training showed no change in startle amplitude when the odor was presented at test. The subsequent experiments in that study showed that the OPS effect was specific to the odor paired with shock at training, and that the effect can be observed at least 28 days after odor-shock pairings and can be achieved using as few as five odor-shock pairings at training.

In the study by Richardson et al. (1999), it was assumed that the OPS effect was mediated by the fear system, given the considerable evidence that FPS to visual and auditory CSs is mediated by fear. The present study was designed to explicitly test this assumption by determining whether systemic injection of a clinical anxiolytic (i.e., diazepam) would attenuate OPS. Systemic administration of diazepam, a GABA-receptor agonist, has previously been shown to attenuate FPS in rats (see Davis et al., 1993) and humans (Patrick, Berthot, \& Moore, 1996). Importantly, diazepam acts in areas of the brain that are known to mediate fear-namely, the central nucleus and basolateral complex of the amygdala (Beck \& Fibiger, 1995; Campeau \& Davis, 1995; Helmstetter, 1993). Furthermore, the results of some studies suggest that the anxiolytic effects of diazepam are not due to any effects that the drug may have on motor activity (e.g., Joordens, Hijzen, \& Olivier, 1998). Hence, if pretest injection of diazepam attenuates the OPS effect, it is likely that OPS is mediated by conditioned fear.

\section{EXPERIMENT 1}

Earlier studies have shown that rats receiving pretest injections of diazepam do not show a potentiated startle response in the presence of a visual CS that has previously been paired with shock (Berg \& Davis, 1984; Davis, 1979). The aim of Experiment 1 was to examine the effect of a 
pretest intraperitoneal injection of diazepam $(0.6 \mathrm{mg} / \mathrm{kg})$ on the potentiated startle response to an olfactory CS that had previously been paired with shock. The dosage of $0.6 \mathrm{mg} / \mathrm{kg}$ of diazepam was chosen on the basis of Berg and Davis' (1984) study, in which this dose of diazepam reduced FPS but had minimal effects on baseline startle amplitude.

\section{Method}

Subjects. Forty experimentally naive Sprague-Dawley male rats, weighing $350-550 \mathrm{~g}$, were used. The rats were obtained from the breeding colony maintained by the School of Psychology at the University of New South Wales. They were housed in groups of 8 in plastic boxes $(65 \times 40 \times 22 \mathrm{~cm})$ with food and water continuously available. The boxes were kept in a colony room with natural lighting. All animals were handled for 5 min per day, for 3 days prior to their being used in the experiment, and all experimental procedures were conducted between $9 \mathrm{a} . \mathrm{m}$. and $5 \mathrm{p} . \mathrm{m}$. American Psychological Association ethical guidelines were followed, and our experimental procedures were approved by the Animal Care and Ethics Committee at the University of New South Wales.

Apparatus. Training and testing occurred in one of two identical startle chambers $(20 \times 12 \times 13 \mathrm{~cm} ; \mathrm{L} \times \mathrm{W} \times \mathrm{H})$. The floor and the two side walls of each chamber were made of stainless steel rods ( $3 \mathrm{~mm}$ in diameter, spaced $13 \mathrm{~mm}$ apart), and the rest of each chamber was made of clear Plexiglas. Each startle chamber was suspended from a sheet of Plexiglas, to which was laminated a strip of piezoelectric film. Movements within the startle chamber caused flexion in the piezoelectric film, which in turn produced a voltage proportional to the size of the movements in the chamber. Thus, larger movements in the chamber resulted in larger voltages. The voltage was amplified and digitized (at a $1-\mathrm{kHz}$ rate) by a custombuilt unit during a $250-\mathrm{msec}$ period that began at the onset of each startle stimulus; the largest response during this period was taken as the subjects' startle amplitude. The startle stimulus was a 100 msec, 100-dB (scale Lin on a type 2335 Brüel \& Kjær sound level meter) white noise burst that had an instantaneous rise-fall time. Two parallel-wired piezoelectric speakers mounted $8 \mathrm{~cm}$ from each side of each startle chamber were used to deliver the startle stimulus. Unscrambled shock ( $0.6 \mathrm{~mA}, 1-\mathrm{sec}$ duration) from a custombuilt constant-current shock generator could be delivered to the floor of each startle chamber. Each startle chamber was housed in a separate light- and sound-attenuating cabinet, in which a $15-\mathrm{W}$ red light provided illumination and a ventilation fan provided $60-\mathrm{dB}$ background noise at all times. The startle chambers were wiped clean with tap water after each training or testing session.

Odor. The odor stimulus consisted of $0.25 \mathrm{ml}$ of undiluted lemon flavor (Natural Lemon, Queen Fine Foods, Australia) placed on a piece of paper towel in a petri dish. The no-odor stimulus consisted of the same materials, except that $0.25 \mathrm{ml}$ of water was used instead of lemon flavor. During the presentation of lemon or water, the petri dish was placed approximately $10 \mathrm{~cm}$ directly below the startle chamber. All preparation of odor stimuli was done in a separate room from that of training and testing.

Procedure. The experiment consisted of a $2 \times 2$ factorial design in which one factor was training (odor-shock paired vs. odor-shock unpaired), and the other was drug treatment at test (diazepam vs. saline). Animals were randomly assigned to four groups so that there were 12 subjects in each paired group and 8 subjects in each unpaired group.

Training. Training consisted of two stages separated by $20-25 \mathrm{~min}$. In the first stage, the rats were placed in the startle chambers and allowed 5 min of adaptation, at the end of which they received the first of five pairings of lemon and shock (paired groups) or water and shock (unpaired groups). On each training trial, the lemon or water petri dish was placed under the startle chamber, and 7-10 sec later the shock was administered. The petri dish was then immediately removed and covered until the next trial. The average time interval between training trials was $2 \mathrm{~min}$. The second stage was identical to the first, except that the paired group received water and no shock and the unpaired group received lemon and no shock. The rats were returned to their home cages between training stages. After each training and testing session, the laboratory windows were opened for 5-10 $\mathrm{min}$ to allow adequate room ventilation.

Testing. One day after training, the rats were placed in the same startle chambers in which they had been trained. After a 5 -min adaptation period, 30 startle stimuli were presented at 30 -sec intervals. No odor was present during this time. The average of the startle responses on Trials 2l-30 was used as an estimate of the baseline startle response amplitude for each animal. Immediately following the 30th startle stimulus, the lemon odor was presented to all animals. Approximately $40 \mathrm{sec}$ after the odor was introduced another 20 startle stimuli were presented at 30 -sec intervals. The odorant remained beneath the startle chambers until the end of the test period. For each subject, the percentage increase in startle was calculated using the following equation: percent increase $=[(M-B) / B] \times 100$, where $M=$ mean startle amplitude in the presence of lemon, and $B=$ baseline startle amplitude. Baseline startle data were analyzed with analysis of variance (ANOVA), and percentage increase in startle at test was analyzed with analysis of covariance (ANCOVA, with baseline as the covariate).

Drugs. Either saline or diazepam was administered intraperitoneally 5 min prior to the commencement of testing - that is 20 min prior to the 21 st startle trial, which was the first of the baseline trials. Diazepam was obtained as Valium (Roche Products, NSW, Australia), which was composed of $5 \mathrm{mg} / \mathrm{ml}$ of diazepam. In order to achieve the final dosage of $0.6 \mathrm{mg} / \mathrm{kg}$, the Valium was diluted 10 min prior to injection with saline such that the resulting concentration of diazepam was $0.6 \mathrm{mg} / \mathrm{ml}$. This was then injected at a volume of $1 \mathrm{ml} / \mathrm{kg}$, as was the saline for the control subjects.

\section{Results and Discussion}

The results showed that diazepam had a marked effect on conditioned OPS and a small effect on baseline responding. The baselines of the diazepam groups (paired = 3,307 arbitrary units $[1-32,000$ range], $S E M=670$; unpaired $=3,704, S E M=1,100$ ) were lower than those of the saline groups (paired $=4,648, S E M=1,219$; unpaired = $5,696, S E M=1,975)$, though the difference was not significant $[F(1,36)=1.8, p=.19]$. The mean percentage increase in startle after odor presentation for each group can be seen in Figure 1. As is evident, diazepam caused a marked reduction in the percent increase of startle in the paired-diazepam group compared with that observed in the paired-saline group. This difference was confirmed by the results of an ANCOVA (with baseline as the covariate), which yielded a significant main effect of both drug treatment $[F(1,35)=7.6, p=.009]$ and training condition $[F(1,35)=4.9, p=.03]$ on percentage increase in startle at test. The interaction of drug treatment and training condition was not significant $[F(1,35)=2.6, p=$ .11]. Subsequent pairwise comparisons, with Tukey's test, showed that the paired-saline group exhibited a larger mean percentage increase in startle than did the other three groups (all $p \mathrm{~s}<.05$ ). No other group differences were significant.

It should be noted that the apparent, but statistically insignificant, difference between drug and saline groups on baseline startle amplitude was due to the high base- 


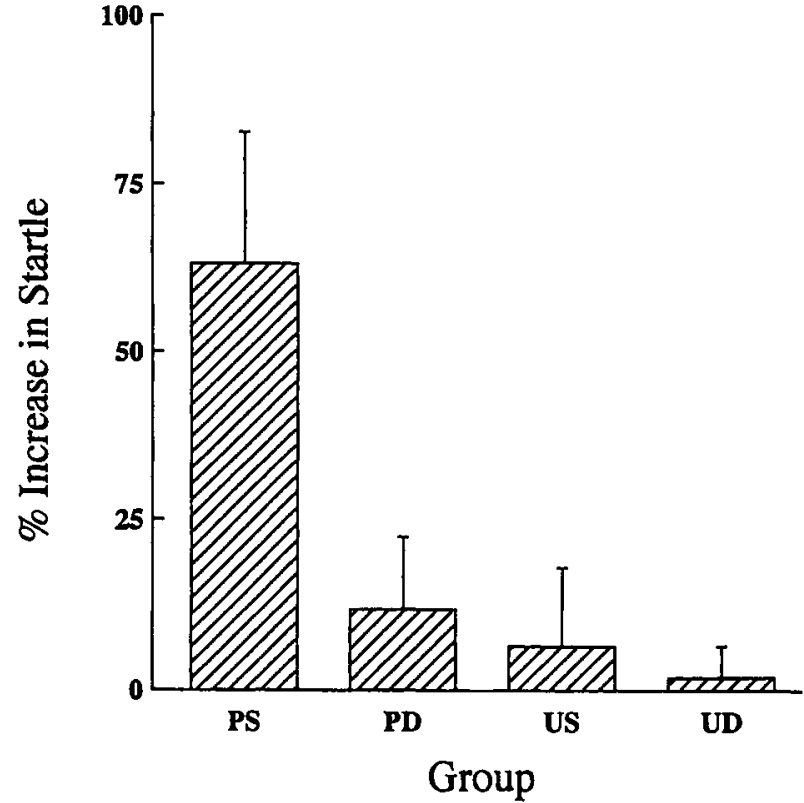

Figure 1. Mean $(+S E M)$ percentage increase in startle from baseline after presentation of lemon odor at test. PS, paired-saline group; PD, paired-diazepam; US, unpaired-saline; UD, unpaireddiazepam.

lines of 2 rats in each of the two saline groups. Deleting the data for these 2 rats in each of the two saline groups results in very comparable baselines between the four groups [paired-diazepam $(n=12)$ : baseline $=3,306$ units; unpaired-diazepam $(n=8)$ : baseline $=3,703$ units; paired-saline $(n=10)$ : baseline $=3,155$ units; unpaired-saline $(n=6)$ : baseline $=3,126]$. Importantly, deleting the data of these rats does not affect the test results. That is, essentially the same mean percent increase in startle is observed at test when all the rats are included in the analysis (see Figure 1) or when the 2 rats in each of the two saline groups with high baselines are deleted (i.e., paired-diazepam $=11 \%$, unpaired-diazepam $=2 \%$, paired-saline $=73 \%$, unpaired-saline $=15 \%$ ). These results show that the statistically nonsignificant difference in baseline startle amplitudes between the drug and saline groups had no influence on the outcome of this experiment.

The results of the present study replicated our results from a previous study in that (1) presentation of the odor at test potentiated startle amplitudes in the paired, but not unpaired groups and (2) the OPS effect was observed after only five odor-shock pairings at training (Richardson et al., 1999). Since the odor (lemon) used in the present study was different from those used in our previous study (grape and eucalyptus), the results also provide further evidence that the OPS effect is not specific to any particular odor.

The present results suggest not only that OPS is mediated by fear, but also that it may be mediated by the same neural substrates that mediate conditioned light potentiated startle. More specifically, the present results are consistent with the previous findings of Berg and Davis (1984) that diazepam attenuated FPS when the CS was in the visual modality. Also consistent with that study was the finding that a dose of $0.6 \mathrm{mg} / \mathrm{kg}$ of diazepam had a small, nonsignificant effect on baseline responding. Since conditioned light potentiated startle is known to be mediated by the basolateral complex (BLA) and central nucleus (Ce) of the amygdala (Campeau \& Davis, 1995), it is also likely that these two structures mediate OPS, given that the BLA and $\mathrm{Ce}$ are the primary neural areas assumed to mediate the anxiolytic effects of systemic diazepam (see, e.g., Beck \& Fibiger, 1995; Helmstetter, 1993). This issue is considered further in the General Discussion section.

Before we can conclude that diazepam attenuates OPS by reducing fear, it is necessary to rule out the possibility that systemic diazepam affects rats' ability to detect an odor. That is, the results of Experiment 1 could be explained by a disruption in odor detection caused by the pretest injection of diazepam. This is unlikely, given that previous studies have shown that diazepam does not significantly alter neural activity in the olfactory bulbs (Beck \& Fibiger, 1995), which are the structures that mediate odor detection. Nonetheless, Experiment 2 was designed to explicitly test whether diazepam would affect the ability to detect lemon odor.

\section{EXPERIMENT 2}

The aim of Experiment 2 was to determine whether diazepam affects rats' ability to detect an odor. Almost all methods used to measure odor detection in rats require either aversive or appetitive conditioning (for a review, see Passe \& Walker, 1985). Such methods could be problematic in the present study since diazepam may potentially act on neural areas that mediate appetitive or aversive responses. Thus, the failure to observe a fear response, for example, may result from the centrally mediated anxiolytic effect of diazepam, rather than effects diazepam may have on peripheral, sensory functions such as odor detection. One way to address this problem is to capitalize on the finding that certain odors elicit unconditional responses in rats, even on the first presentation. For instance, pilot studies in our laboratory showed that when placed in a closed compartment, rats avoided the side of the compartment that was scented with lemon. Given that lemon odor has no apparent significance to the survival of the species, it is unlikely that the avoidance is mediated by unconditioned fear. Rather, it is more likely that rats avoid the citrus odor because it is unpleasant. Hence, using an unconditioned-avoidance procedure, we designed Experiment 2 to determine whether intraperitoneal injection of diazepam would disrupt odor detection. If diazepam does diminish rats' ability to detect the odor, a group given pretest diazepam should avoid lemon odor less than should a saline control group. 


\section{Method}

Subjects. Thirty-two experimentally naive, Sprague-Dawley male rats, weighing $350-550 \mathrm{~g}$, were used as subjects. The rats were obtained from the same source described in Experiment 1 and were maintained and handled as in Experiment 1 .

Apparatus. Testing occurred in a black/white shuttle box. The box was made of Plexiglas $(40 \times 19 \times 20 \mathrm{~cm} ; \mathrm{L} \times \mathrm{W} \times \mathrm{H})$ and was divided by a wall into two identical chambers, each $20 \mathrm{~cm}$ in length. The dividing wall had a small square opening ( $8-\mathrm{cm}$ side length) at floor level, to allow animals to pass between chambers. This opening could be blocked by a guillotine door. The floor of the box consisted of metal bars (2-mm diameter, $1 \mathrm{~cm}$ center to center) and was raised such that the entire box stood $11 \mathrm{~cm}$ above an underlying litter tray. The top of each chamber was a hinged door, which could be opened independently. The walls of one of the chambers were painted white, and its top was clear Plexiglas; the other chamber (including the top) was painted black. Importantly, the only illumination was provided by a $40-\mathrm{W}$ red light, positioned approximately $15 \mathrm{~cm}$ above the center of the box, so that the animals could not distinguish between chambers on the basis of color or illumination.

Odor. Odor stimuli consisted of the same materials as described in Experiment 1. During odor presentation, the petri dish was placed $5 \mathrm{~cm}$ below the center of the chamber.

Procedure. The experiment consisted of a $2 \times 2$ design in which one factor was odor (lemon vs. water) and the other was drug injection (diazepam vs. saline). The animals were randomly allocated into groups, each containing 8 rats. To familiarize the animals with the apparatus, they were placed in each chamber for $2 \mathrm{~min}$, approximately $1 \mathrm{~h}$ before testing. During this time, the door connecting the chambers was closed. They were then returned to their home cage for approximately $40 \mathrm{~min}$. Twenty minutes before test the rats were given an intraperitoneal injection of either saline or diazepam $(0.6 \mathrm{mg} / \mathrm{kg})$. At test, the odor stimulus (either lemon or water) was placed under the black chamber (i.e., the odor chamber). During the initial placement of the odor stimulus, the top of the odor chamber was closed, and the top in the other (no-odor) chamber was open, as was the passage joining the two chambers. Approximately $15 \mathrm{sec}$ after introduction of the odor stimulus, the animal was placed in the no-odor chamber, and its top was closed. The rat was left in the box for $4 \mathrm{~min}$, during which the time spent in the odor chamber was recorded. Four subjects from each saline group and 8 from each diazepam group were also scored for the number of crosses between chambers. The odor stimulus remained in place until the end of the test. After each animal was tested, the apparatus was cleaned with tap water, and the chamber tops were opened for $5 \mathrm{~min}$, during which time the laboratory windows were also open. Differences between groups in time spent in the odor chamber, and in crosses between chambers, were analyzed with an ANOVA.

\section{Results and Discussion}

The rats in the lemon groups spent considerably less time in the odor chamber than did the rats in the water groups (Figure 2); this difference was confirmed by a significant main effect of odor $[F(1,28)=38.5, p=.001]$. There was, however, no significant main effect of drug $(F<1)$, nor was there a significant interaction $(F<1)$. There were also fewer crosses between chambers in the lemon groups (saline: 7.5 crosses, $S E M=1.3$, diazepam: $9.1, S E M=1.6$ ) than in the water groups (saline: 10 , $S E M=1.3$, diazepam: $12.4, S E M=1.0$ ), with the difference approaching significance $[F(1,20)=3.6, p=$ .07]. However, neither the main effect of drug condition on the number of crosses between chambers $[F(1,20)=$

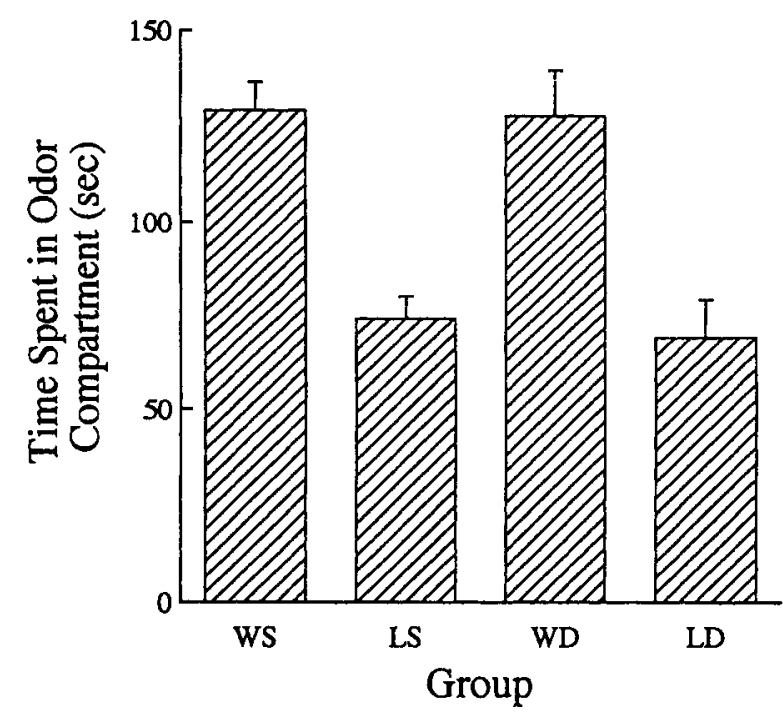

Figure 2. Average ( $+S E M)$ time spent in odor chamber either with odor (lemon) or without odor (water) over a 4-min test period. WS, water-saline; LS, lemon-saline; WD, water-diazepam; LD, lemon-diazepam.

$1.7, p=.2]$, nor the interaction of drug condition and odor $(F<1)$ was significant.

The main effect of odor on time spent in the odor chamber shows that rats avoided the chamber scented with lemon odor. The similar performance of the lemondiazepam and lemon-saline groups suggests not only that the avoidance was not mediated by fear, but more importantly, that this dose of systemic diazepam does not diminish rats' ability to detect the lemon odor. In addition, diazepam did not significantly affect the number of crosses between chambers, suggesting that the dose administered did not affect the rats' mobility.

\section{GENERAL DISCUSSION}

The results of Experiment 1 replicated the results of the previous study by Richardson et al. (1999) in that an odor previously paired with shock enhanced rats' startle responses. Furthermore, a low dose $(0.6 \mathrm{mg} / \mathrm{kg})$ of diazepam administered intraperitoneally prior to test was sufficient to attenuate conditioned OPS. The results of Experiment 2 suggest that the effect of systemic, diazepam on OPS was not due to a disruption in the ability to detect the odor or to a reduction in mobility. Given the known anxiolytic effects of diazepam, the results of the present study provide evidence that OPS is mediated by the fear system.

As mentioned earlier, the results of this study are similar to those of an earlier study by Berg and Davis (1984), in which a visual CS was used to potentiate the startle response. In addition to their finding that systemic diazepam attenuated FPS, they also showed that this attenuation 
was abolished by an additional injection of RO 15-1788, a specific benzodiazepine antagonist. This suggests that in their study, and most likely in the present study, the effects of systemic diazepam were mediated by benzodiazepine receptors. One area that has a high density of benzodiazepine receptors is the basolateral complex of the amygdala (BLA), and the results of some studies suggest that the BLA is a site at which benzodiazepine agonists exert their anxiolytic effects. For instance, local infusion of midazolam into the BLA significantly increases open-arm activity in the elevated plus maze (Green \& Vale, 1992). Sanders and Shekhar (1995) also reported that infusion of flumazenil, a benzodiazepine receptor antagonist, into the BLA of rats reversed the anxiolytic effects of systemic chlordiazepoxide in the social interaction test. In addition, local infusion of diazepam in the BLA has been shown to attenuate both freezing and hypoalgesia following footshocks (Helmstetter, 1993). Taking this research into account, the results of the present study suggest that OPS may be mediated by benzodiazepine receptor activity in the BLA, though more direct evidence is required.

Despite the paucity of relevant studies, it is apparent that the neural mechanisms that have been proposed to mediate conditioned fear of simple auditory and visual stimuli may also be involved in conditioned olfactory fear. A considerable literature suggests that the BLA and $\mathrm{Ce}$ of the amygdala are crucial mediators of fear responses to visual and auditory CSs (for a review, see Davis et al., 1993). Cousens and Otto (1998) have recently found that rats that had received either pre- or posttraining lesions of the BLA also show a marked reduction in freezing to an olfactory CS. Furthermore, Sananes and Campbell (1989) reported that lesions of the Ce in 21 -day-old rats disrupted conditioned heart-rate responses to an odor that had previously been paired with shock. These results support a general model of the neural underpinnings of conditioned fear, such as that proposed by Davis (1992), in which the amygdala receives information from the auditory, visual, and olfactory modalities and coordinates appropriate fear responses.

It is, however, still uncertain as to how olfactory information reaches the amygdala from the olfactory bulbs. One possible route may be via the olfactory amygdala (see De Olmos, Alheid, \& Beltramino, 1985), a group of nuclei in the ventral part of the amygdala that receive direct projections from the olfactory bulbs and have reciprocal connections with the olfactory cortex. A number of nuclei of the olfactory amygdala send projections to either the BLA, Ce, or both, and may be involved in processing olfactory information in regard to potential physical threat to the animal. By studying immediate-early gene expression, we are currently examining the role of the olfactory amygdala, BLA, and Ce in fear responses to conditioned odors.

\section{REFERENCES}

Beck, C. H. M., \& Fibiger, H. C. (1995). Conditioned fear-induced changes in behavior and in the expression of the immediate early gene c-fos: With and without diazepam pretreatment. Journal of Neuroscience, 15, 709-720.

BERG, K. W., \& DAVIS, M. (1984). Diazepam blocks fear-enhanced startle elicited electrically from the brainstem. Physiology \& Behavior, 32, 333-336.

Campeau, S., \& Davis, M. (1995). Involvement of the central nucleus and basolateral nucleus of the amygdala in fear conditioning measured with fear potentiated startle in rats trained concurrently with auditory and visual conditional stimuli. Journal of Neuroscience, 106, 569-574.

Charney, D. S., Grillon, C., \& Bremner, J. D. (1998). The neurobiological basis of anxiety and fear: Circuits, mechanisms, and neurochemical interactions (Pt. 1). Neuroscientist, 4, 35-44.

Cousens, G., \& Otto, T. (1998). Both pre- and posttraining excitotoxic lesions of the basolateral amygdala abolish the expression of olfactory and contextual fear conditioning. Behavioral Neuroscience, 112 , 1092-1103.

Davis, M. (1979). Diazepam and Flurazepam: Effects on conditioned fear as measured with the potentiated startle paradigm. Psychopharmacology, 62, 1-7.

DAvis, M. (1992). The role of the amygdala in fear and anxiety. Annual Review of Neuroscience, 15, 353-375.

Davis, M., Falls, W. A., Campeau, S., \& Kim, M. (1993). Fear potentiated startle: A neural and pharmacological analysis. Behavioural Brain Research, 58, 175-98

De Olmos, J., Alheid, G. F., \& Beltramino, C. A. (1985). Amygdala. In G. Paxinos (Ed.), The rat nervous system (pp. 223-334). Sydney: Academic Press.

GreEN, S., \& VALE, A. L. (1992). Role of amygdaloid nuclei in the anxiolytic effects of benzodiazepines in rats. Behavioural Pharmacology, 3, 261-264.

Helmstetter, F. J. (1993). Stress-induced hypoalgesia and defensive freezing are attenuated by application of diazepam to the amygdala. Pharmacology, Biochemistry \& Behavior, 44, 433-438.

JoOrdens, R. J. E., HiJzen, T. H., \& Olivier, B. (1998). The anxiolytic effect on the fear-potentiated startle is not due to a non-specific disruption. Life Sciences, 63, 2227-2232.

Leaton, R. N., \& Borszcz, G. S. (1985). Potentiated startle: Its relation to freezing and shock intensity in rats. Journal of Experimental Psychology: Animal Behavior Processes, 11, 421-428.

LEDoux, J. E. (1993). Emotional memory systems in the brain. Behavioural Brain Research, 58, 69-79.

PASSE, D. H., \& WALKER, J. C. (1985). Odor psychophysics in vertebrates. Neuroscience \& Biobehavioral Reviews, 9, 431-467.

Patrick, C. J., Berthot, B. D., \& Moore, J. D. (1996). Diazepam blocks fear-potentiated startle in humans. Journal of Abnormal Psychology, 105, 89-96.

Richardson, R., Vishney, A., \& LeE, J. (1999). Conditioned odorpotentiation of startle in rats. Behavioral Neuroscience, 113, 787-794.

Sananes, C. B., \& Campbell, B. A. (1989). Role of the central nucleus of the amygdala in olfactory heart rate conditioning. Behavioral Neuroscience, 103, 519-525.

Sanders, S. K., \& Shekhar, A. (1995). Anxiolytic effects of chlordiazepoxide blocked by injection of GABA-sub(A) and benzodiazepine receptor antagonists in the region of the anterior basolateral amygdala of rats. Biological Psychiatry, 37, 473-476

(Manuscript received March 1, 2000; accepted for publication March 1, 2000.) 